\title{
Peritoneal dialysis as a life-saving procedure in an extremely low birth weight infant: case report and review of the literature
}

\author{
Merih Çetinkaya $^{1 \oplus}$, Tuğba Erener Ercan ${ }^{2 \oplus}$, Sevgi Yavuz $^{3 \oplus}$, Seyithan Özaydın ${ }^{4 \oplus}$ \\ Departments of ${ }^{1}$ Neonatology, ${ }^{3}$ Pediatric Nephrology and ${ }^{4}$ Pediatric Surgery, Health Sciences University, Kanuni Sultan Suleyman \\ Training and Research Hospital, Istanbul; ${ }^{2}$ Department of Neonatology, Maltepe University, Faculty of Medicine, Istanbul, Turkey.
}

\begin{abstract}
Background. Acute kidney injury (AKI) is a common condition in the neonatal intensive care unit (NICU), particularly in preterm infants. Management of AKI in neonates is challenging. Peritoneal dialysis (PD) has been preferred as the most applicable modality in neonates when medical therapy fails.

Case. A female infant was born at 24 and 4/7 weeks with a birth weight of 460 grams after an emergency cesarian section from a preeclamptic pregnacy. She developed AKI secondary to sepsis. A neonatal, straight single-cuff Tenckhoff catheter was inserted and PD was started on day 12. PD was discontinued after 6 days, on day 18 with adequate urine output and normalization of kidney function tests. However, the patient died on day 152 secondary to a nosocomial infection.

Conclusion. To the best of our knowlegde, our case is the smallest infant in whom PD was performed succesfully with a PD catheter. PD is a relatively safe, effective and a feasible therapy in the neonatal population even in the smallest infants. PD may be a live-saving procedure in extremely low birth weight infants with severe AKI.
\end{abstract}

Key words: acute kidney injury, extremely low birth weight, peritoneal dialysis, preterm.

Acute kidney injury (AKI) is defined as a decrease in kidney function associated with an increase in serum creatinine (Scr) levels which may or may not be accompanied by a reduction in urine output. ${ }^{1,2}$ AKI has a great impact on morbidity and mortality in critically ill children and those who survive an episode of AKI should have long term follow up with regard to the risk of development of chronic kidney diease. ${ }^{3,4}$ Among the neonatal population, AKI although still an underrecognized morbidity, is a common condition in the neonatal intensive care unit (NICU), particularly in preterm infants. It occurs in 40 to $70 \%$ of critically ill neonatal intensive care admissions., ${ }^{5,6}$ Premature infants, especially those with lower

\footnotetext{
Tuğba Erener Ercan

terenerercan@gmail.com
}

Received 18th July 2019, revised 2nd December 2019, accepted 13th April 2020. birth weights are susceptible to AKI given to the incomplete nephrogenesis and their lower nephron number. ${ }^{6,7}$ AKI has been reported to be associated with morbidity, longer hospital stays and mortality in this vulnerable population. ${ }^{2,4,6,8}$

Management of AKI in neonates is challenging. Prevention of AKI should be the first priority in management. However, data are sparse with regard to interventions to prevent AKI in at risk patients or to improve the outcome once it is established. ${ }^{4,9}$ When medical management of AKI fails, the primary therapy is renal replacement therapy (RRT). Although indications for RRT are not very well described among neonates with AKI; refractory acidosis, uremia, electrolye disturbances, inability to provide adequate nutrition and fluid overload are the most common indications. Peritoneal dialysis (PD), intermittent hemodialysis (HD) and continuous renal replacement therapy (CRRT) are three common methods of RRT 
in neonates. ${ }^{4,7} \mathrm{PD}$ has been preferred as the most applicable modality in neonates so far. ${ }^{7,10}$ However, its use has usually been deferred during the course of AKI especially in extremely low birth weight infants (ELBW) due to the fear of technical challenges. ${ }^{9}$ In fact, several studies have described successful application of PD in critically ill ELBW infants. ${ }^{11-21}$ Herein, we described our experience of PD in an ELBW infant who developed AKI secondary to sepsis.

\section{Case Report}

A female infant was born at 24 and $4 / 7$ weeks with a birth weight of 460 grams after an emergency cesarian section. The pregnancy was complicated by preeclampsia. The apgar scores were 3 and 7 at 1 and 5 minutes, respectively. The patient was intubated in the delivery room and was given surfactant in the NICU due to the diagnosis of respiratory distress syndrome and conventional mechanical ventilation was started. The patient developed stage 4 intraventricular hemorrhage on day- 3 and she was given an erythrocyte infusion. On day-10, she became hypotensive secondary to sepsis which was refractory to fluid resuscitation and she was given inotrope treatment. On day11 , the infant became anuric with worsening kidney function tests. Anuria was unresponsive to furosemide and Scr and blood urea nitrogen (BUN), were found to be $1.59 \mathrm{mg} / \mathrm{dl}$ and $50 \mathrm{mg} /$ $\mathrm{dl}$, respectively. Serum $\mathrm{Na}, \mathrm{K}, \mathrm{Ca}$, phosphorus and uric acid levels were $184 \mathrm{meq} / \mathrm{l}, 5.9 \mathrm{meq} / \mathrm{l}$, $10.3 \mathrm{mg} / \mathrm{dl}, 3.3 \mathrm{mg} / \mathrm{dl}$ and $7.8 \mathrm{mg} / \mathrm{dl}$, respectively. On day-12, the patient was still anuric and became edematous with a $15.2 \%$ fluid weight gain from birth. She was transitioned to high frequency oscillatory ventilation from conventional ventilation due to her worsening respiratory status secondary to fluid overload. Given the worsening respiratory and metabolic acidosis (venous pH: 7.20, $\mathrm{PCO} 2: 132 \mathrm{mmHg}$, PO2: $22.4 \mathrm{mmHg}$, HCO3: $12.4 \mathrm{mmol} / \mathrm{l}, \mathrm{BE}:-$ 11.7 and oxygen saturation $\% 88$ ), electrolyte imbalance and generalized edema, PD was started on day-12. A neonatal, straight $10 \mathrm{Fr}$ single-cuff Tenckhoff catheter (Cook Medical,
Bloomington, USA) with a length of $8 \mathrm{~cm}$ was inserted with a left paramedian entry site above the umbilicus at the bedside by a pediatric surgeon. Cutaneous and subcutaneous tissues were dissected down to the sheath of the rectus abdominal muscle and the guided PD catheter was inserted and advanced along the peritoneum, and directed to the left lower quadrant of the abdomen with the distal end placed in pelvis. Dialysis was started with a fillvolume of $20 \mathrm{ml} / \mathrm{kg}$ for 12 cycles per day. The fill and dwell times were 10 and 60 minutes, respectively. Biocompatible $\mathrm{PD}$ solution (glucose 1.36\%, Na $132 \mathrm{mmol} / \mathrm{L}, \mathrm{Cl} 95 \mathrm{mmol} / \mathrm{L}$, Ca $1.25 \mathrm{mmol} / \mathrm{L}, \mathrm{HCO} 325 \mathrm{mmol} / \mathrm{L}$, laktat 15 $\mathrm{mmol} / \mathrm{L}$, ph 7.40) was used. On the $3^{\text {rd }}$ day of succesful PD (day-15), she was again placed on conventional ventilation which she tolerated well. Leakage of peritoneal fluid from the exit site was the only complication noted during the dialysis procedure. However, it did not impair PD efficacy and the infant's condition improved with decreasing Scr and BUN levels $(0.77 \mathrm{mg} / \mathrm{dl}$ and $47.6 \mathrm{mg} / \mathrm{dl}$, respectively) and a urine output of $3.6 \mathrm{ml} / \mathrm{kg} /$ day (24 hours of PD). PD was discontinued after 6 days, on day-18. Adequate urine output was maintained without the need for diuretics and renal function tests improved to a BUN level of $20 \mathrm{mg} / \mathrm{dl}$ and a Scr level of $0.57 \mathrm{mg} / \mathrm{dl}$ and the serum levels of $\mathrm{Na}, \mathrm{K}$ and HCO3 were $140 \mathrm{meq} / \mathrm{l}, 4 \mathrm{meq} / \mathrm{l}$ and $31 \mathrm{mmol} / \mathrm{l}$, respectively with supplementation of $4 \mathrm{meq} /$ $\mathrm{kg}$ of $\mathrm{Na}$ and $2 \mathrm{meq} / \mathrm{kg}$ of $\mathrm{K}$ in total parenteral solution without any $\mathrm{HCO} 3$ supplementation at this stage. No particular medication was given for renal dysfunction. However, the dosage of antibiotics was re-arranged according to the estimated creatinine clearance. Patient's Scr and BUN levels remained stable during the rest of her hospital stay and the lowest serum creatinine level which was $0.3 \mathrm{mg} / \mathrm{dl}$ was detected at day 90 when she was 1715 grams in weight. Renal ultrasonography was performed three times during her hospital stay and all revealed normal ultrasonographic findings without any increase in renal echogenicity and pelvicalyceal dilatation. The patient was never discharged and died on day 152 secondary to a nosocomial 
infection which led to worsening symptoms of severe bronchopulmonary dysplasia (BPD) and severe pulmonary hypertension (PHT). No autopsy was performed.

Informed consent was received from the family before the preparation of the manuscript.

\section{Discussion}

Preterm infants are susceptible to perinatal and nosocomial infections, hemodynamic alterations, nephrotoxic medications which make them vulnerable to impairement of kidney function besides their physiologic vulnerability given their incomplete nephrogenesis and low nephron number. ${ }^{6,7,22,23}$ By using contemporary definitions based on the neonatal modified KDIGO criteria (Table I), the incidence of AKI in low birth weight infants ranges between 19 to $40 \% .8,24,25$

Sepsis is also an important risk factor for AKI among neonates. Among preterm infants, incidence of AKI secondary to sepsis has been postulated to be as high as $75.6 \% .^{24}$ Sepsis has been reported to contribute to AKI secondary to the hypotension associated with systemic inflammation. However, it may directly damage the kidney by effects on microvasculature. ${ }^{4,27-30}$

Our case developed AKI secondary to sepsis. She carried the reported risk factors for AKI such as being an ELBW premature infant and having sepsis which led to hypotension refractory to inotrope support. RRT was considered in our case due to acidosis, uremia and anuria refractory to diuretic therapy which led to significant fluid overload. Providing RRT is the expected standard of care for neonates developing AKI when it is indicated. ${ }^{10}$ To our knowledge, our case report represents the smallest infant in whom PD was successful using a standard PD catheter. The smallest infant who was treated with PD reported to date was from Japan who was 24 weeks and 3 days of gestation weighing 264 grams. ${ }^{16}$ However, in that male infant a drainage tube was used for PD instead of a standard PD catheter and PD was continued for 32 days. In the study of Stojanovic et al. ${ }^{19}$ a large-bore intravenous cannula was used for PD in a 27 week old preterm infant of 470 grams. In the present case, Tenckhoff PD catheter placed in the lower left quadrant was used and dialysis was started with fill volumes of $20 \mathrm{ml} / \mathrm{kg}$. Harshman et al. ${ }^{11}$ also used a commercially available PD catheter which was inserted in the left upper quadrant in an ELBW infant (830 grams) for PD with initial fill volumes of $20 \mathrm{ml} / \mathrm{kg}$. In the case series of Macchini et al. ${ }^{17}$ Tenchoff catheters were used for two ELBW (630 and 700 grams) infants for PD. In the study of Ustyol et al. ${ }^{18}$ Tenckhoff catheters placed in the left lower quadrant were used for PD in 31 neonates, 16 of whom were preterm infants with the lowest gestational age (GA) and birth weight (BW) of 24 weeks and 580 grams, respectively. In other two large case series, PD was performed in a group of preterm infants with a minimum GA and BW of 27 weeks and 1000 gr, respectively using PD catheters in the infra-umbilical position. ${ }^{12,13}$ Our case report was unique for a succesfull PD by using a commercially avalaible PD catheter with the placement of the distal end in the pelvis in

Table I. Neonatal KDIGO (Kidney Diseases: Improving Global Outcomes) acute kidney injury definition.

\begin{tabular}{lll}
\hline Stage & Serum creatinine $(\mathrm{Scr})$ & Urine output over 24 hours \\
\hline 0 & No change in serum creatinine or rise $<0.3 \mathrm{mg} / \mathrm{dL}$ & $>1 \mathrm{~mL} / \mathrm{kg} / \mathrm{h}$ \\
1 & SCr rise $\geq 0.3 \mathrm{mg} / \mathrm{dL}$ within $48 \mathrm{~h}$ or $\mathrm{SCr}$ rise $\geq 1.5$ to $1.9 \mathrm{x}$ reference $\mathrm{SCr} \mathrm{r}^{\mathrm{a}}$ & $>0.5$ and $\leq 1 \mathrm{~mL} / \mathrm{kg} / \mathrm{h}$ \\
2 & within 7 days & $>0.3$ and $\leq 0.5 \mathrm{~mL} / \mathrm{kg} / \mathrm{h}$ \\
3 & SCr rise $\geq 2$ to $2.9 \times$ reference $S C r^{\mathrm{a}}$ & $\leq 0.3 \mathrm{~mL} / \mathrm{kg} / \mathrm{h}$ \\
\hline
\end{tabular}

a Reference $\mathrm{SCr}$ will be defined as the lowest previous $\mathrm{SCr}$ value.

b SCr value of $2.5 \mathrm{mg} / \mathrm{dL}$ represents $<10 \mathrm{~mL} / \mathrm{min} / 1.73 \mathrm{~m}^{2}$. 


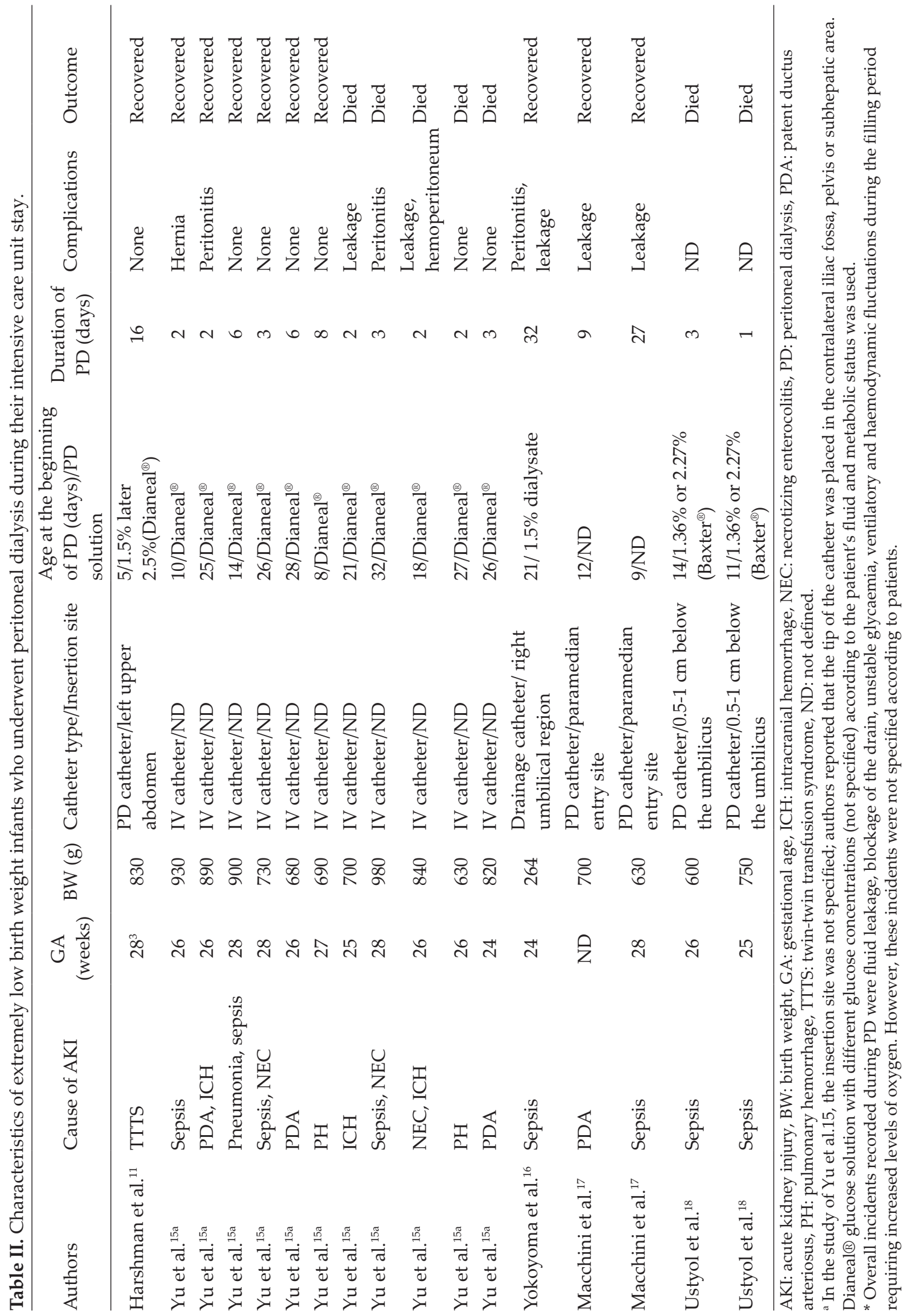




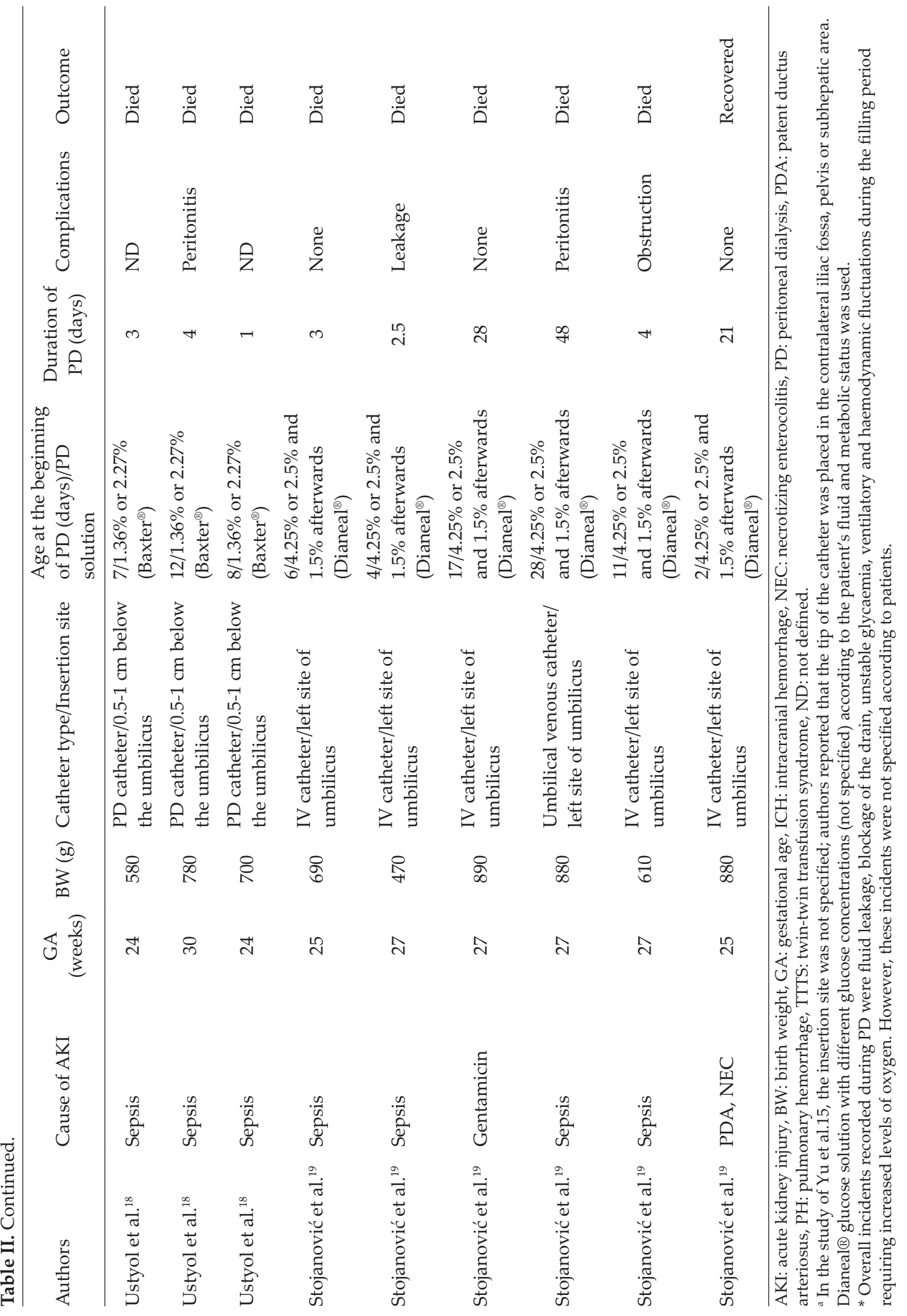




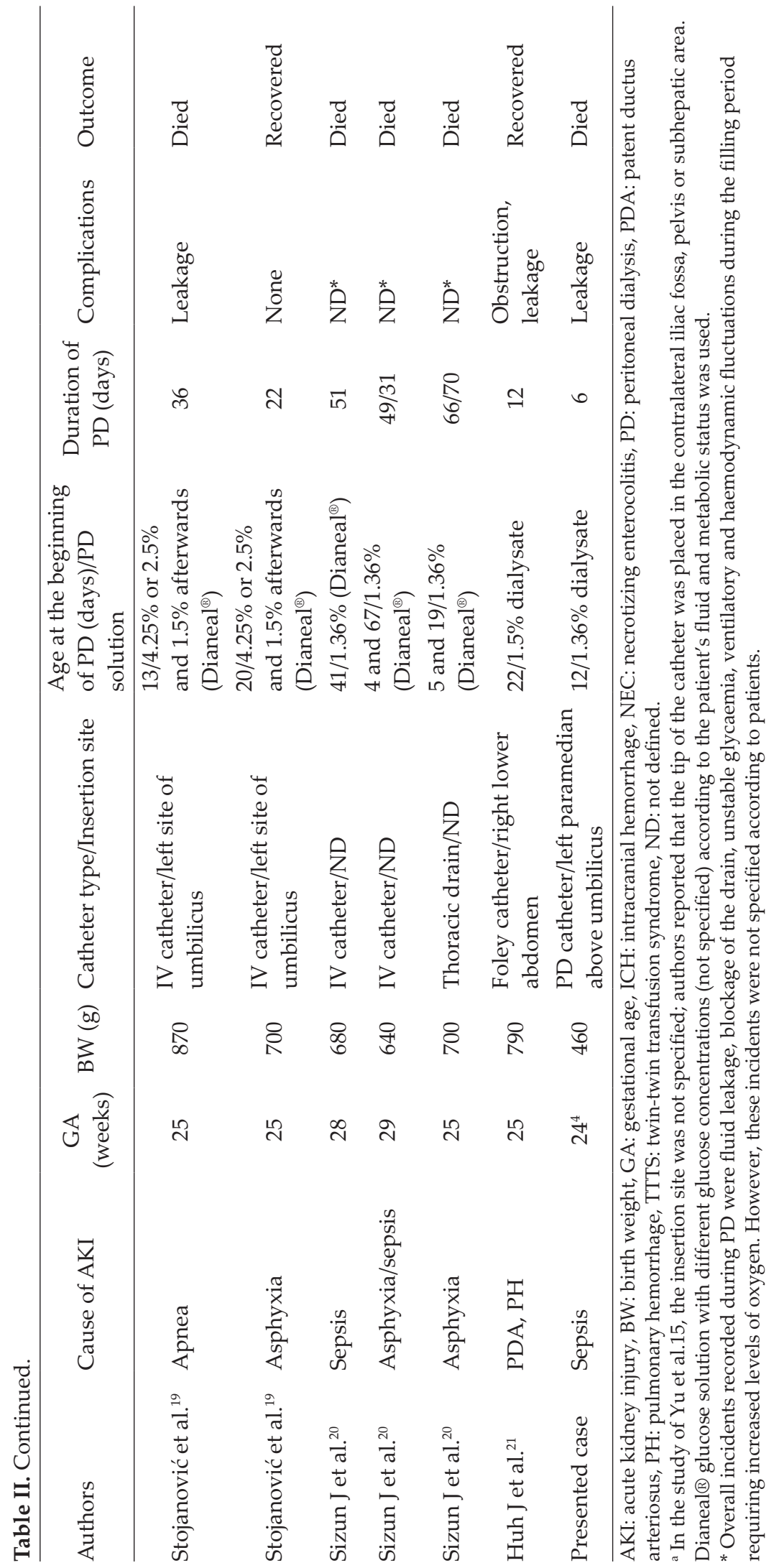


an extremely preterm infant with an ELBW. In Table II, a brief review of characteristics of ELBW infants described in the literature who underwent PD during their NICU stay was presented.

Acute PD has been reported to have a high incidence of technical problems in small infants, specifically leakage of peritoneal fluid around the catheter entry site. ${ }^{9}$ In a retrospective study of Maizlin et al. ${ }^{31}$ where outcome assessment of RRT in neonates were reviewed during an eight year period, the most frequently experienced complications in PD patients were reported to be related to peritonitis $(83 \%)$, catheter malfunctions (72\%) and PD catheter leaks (55\%). Hakan et al. ${ }^{32}$ reported their 7 year experience of acute PD in 77 neonates and complications of procedure were noted as hyperglycemia $(n=35)$, leaking of dialysate $(n=13)$, peritonitis $(n=10)$, catheter obstruction $(n=3)$, bleeding when inserting the catheter $(n=3)$, exit site infection $(n=2)$, and bowel perforation $(n=1)$. In the above mentioned studies of Ustyol et al. ${ }^{18}$, Alparslan et al. ${ }^{12}$ and Unal et al. ${ }^{13}$ the rate of PD related complications were reported to be $48.4 \%, 25.9 \%$ and $40 \%$, respectively with leakage, occlusion and infection being the most commonly observed complications. In the present case, minimal leakage occurred around catheter entry site which did not impair the efficacy of PD and no revision of the catheter was needed. Leakage from the catheter entry site which is one of the most common complications of PD has been attributed to the inelastic nature of the premature infants' skin which forms a poor seal. ${ }^{9}$

The prognosis of AKI in neonates is mostly dependent on the underlying condition and on gestational age. Among very low birth weight (VLBW) and ELBW neonates, AKI was found to be independently associated with increased mortality. ${ }^{1,4,25}$ High mortality rates have also been reported in neonates treated with PD although these mortality rates were usually attributed to the underlying etiology and being premature. ${ }^{12,13.31,32}$ The present case died on day
152 of life secondary to a nosocomial infection with worsening symptoms of BPD and PHT. She survived the acute episode of kidney injury with succesfull application of PD.

In a recent survey, it was reported that neonatal AKI was underappreciated, particularly among neonatologists due to a lack of evidence on neonatal AKI. ${ }^{5}$ It should be kept in mind that AKI in neonates is common, particularly in neonates with multiple risk factors such as prematurity, sepsis and use of nephrotoxic drugs. ${ }^{7}$ Acute PD has several advantages for neonates, particularly for low birth weight neonates due to the relative technical ease of insertion in the setting of NICU and lack of the most challenging technical problems with regard to need for vascular access and an exracorporeal blood circuit or renal support therapy machines as in the case of HD and CRRT. Therefore, PD can be a rescue therapy for ELBW infants with AKI. Our patient represented a good example for a rescue therapy of PD as one of the smallest infants in the literature during the first weeks of life who later maintained normal renal function.

In conclusion PD is a relatively safe, effective and a feasible therapy in the neonatal population even in the smallest infants. To the best of our knowlegde, our case is the smallest infant in whom PD was performed succesfully with a PD catheter. This also suggests that $\mathrm{PD}$ may be a live-saving procedure in ELBW infants with severe AKI. In an era in which neonates are routinely supported with many invasive procedures, we should not be reluctant to initiate RRTs with the fear of their small sizes and lower gestational ages which could give them a chance of survival and an improvement in outcome.

\section{REFERENCES}

1. Sweetman DU. Neonatal acute kidney injurySeverity and recovery prediction and the role of serum and urinary biomarkers. Early Hum Dev 2017; 105: 57-61.

2. Jetton JG, Askenazi DJ. Acute kidney injury in the neonate. Clin Perinatol 2014; 41: 487-502. 
3. Sutherland SM, Ji J, Sheikhi FH, et al. AKI in hospitalized children:epidemiology and clinical associations in a national cohort. Clin J Am Soc Nephrol 2013; 8: 1661-1669.

4. Selewski DT, Charlton JR, Jetton JG, et al. Neonatal acute kidney injury. Pediatrics 2015; 136: e463-e473.

5. Kent AL, Charlton JR, Guillet $R$, et al. Neonatal acute kidney injury: a survey of neonatologists' and nephrologists' perceptions and practice management. Am J Perinatol 2018; 35: 1-9.

6. Lee CC, Chan OW, Lai MY, et al. Incidence and outcomes of acute kidney injury in extremely-lowbirth-weight infants. PLoS One 2017; 12: e0187764.

7. Nada A, Bonachea EM, Askenazi DJ. Acute kidney injury in the fetus and neonate. Semin Fetal Neonatal Med 2017; 22: 90-97.

8. Koralkar R, Ambalavanan N, Levitan EB, McGwin G, Goldstein S, Askenazi D. Acute kidney injury reduces survival in very low birth weight infants. Pediatr Res 2011; 69: 354-358.

9. Coulthard MG. The management of neonatal acute and chronic renal failure: a review. Early Hum Dev 2016; 102: 25-29.

10. Kaddourah A, Goldstein SL. Renal replacement therapy in neonates. Clin Perinatol 2014; 41: 517-527.

11. Harshman LA, Muff-Luett M, Neuberger ML, et al. Peritoneal dialysis in an extremely low-birth weight infant with acute kidney injury. Clin Kidney J 2014; 7: 582-585.

12. Alparslan C, Yavascan O, Bal A, et al. The performance of acute peritoneal dialysis treatment in neonatal period. Ren Fail 2012; 34: 1015-1020.

13. Unal S, Bilgin L, Gunduz M, Uncu N, Azili MN, Tiryaki T. The implementation of neonatal peritoneal dialysis in a clinical setting. J Matern Fetal Neonatal Med 2012; 25: 2111-2114.

14. Oyachi N, Obana K, Kimura S, Kubo M, Naito A, Nemoto A. Use of a flexible Blake ${ }^{\circledR}$ silicone drains for peritoneal dialysis in the neonatal intensive care unit. Pediatr Int 2011; 53: 417-418.

15. Yu JE, Park MS, Pai KS. Acute peritoneal dialysis in very low birth weight neonates using a vascular catheter. Pediatr Nephrol 2010; 25: 367-371.

16. Yokoyama S, Nukada T, Ikeda Y, Hara S, Yoshida A. Successful peritoneal dialysis using a percutaneous tube for peritoneal drainage in an extremely low birth weight infant: a case report. Surg Case Rep 2017; 3: 115.

17. Macchini F, De Carli A, Testa S, et al. Feasibility of peritoneal dialysis in extremely low birth weight infants. J Neonatal Surg 2012; 1: 52.
18. Ustyol L, Peker E, Demir N, Agengin K, Tuncer O. The use of acute peritoneal dialysis in critically Ill newborns. Med Sci Monit 2016; 22: 1421-1426.

19. Stojanović VD, Bukarica SS, Antić JB, Doronjski AD. Peritoneal dialysis in very low birth weight neonates. Perit Dial Int 2017; 37: 389-396.

20. Sizun J, Giroux JD, Rubio S, Guillois B, Alix D, De Parscau L. Peritoneal dialysis in the very low-birthweight neonate (less than 1000 g). Acta Paediatr 1993; 82: 488-489.

21. Huh J, Hwang J, Lee EH, Boo YJ, Choi BM, Hong YS. Successful peritoneal dialysis in an extremely preterm infant. Neonatal Med 2016; 23: 158-162.

22. Cataldi L, Leone R, Moretti U, et al. Potential risk factors for the development of acute renal failure in preterm newborn infants:a case control study. Arch Dis Child Fetal Neonatal Ed 2005; 90: F514-F519.

23. Rodriguez-Soriano J, Aguirre M, Oliveros R, Vallo A. Long-term renal follow-up of extremely low birth weight infants. Pediatr Nephrol 2005; 20: 579-584.

24. Jetton JG, Askenazi DJ. Update on acute kidney injury in the neonate. Curr Opin Pediatr 2012; 24: 191-196.

25. Carmody JB, Swanson JR, Rhone ET, Charlton JR. Recognition and reporting of AKI in very low birth weight infants. Clin J Am Soc Nephrol 2014; 9: 20362043.

26. El-Badawy AA, Makar S, Abdel-Razek AR, Abd Elaziz D. Incidence and risk factors of acute kidney injury among the critically ill neonates. Saudi J Kidney Dis Transpl 2015; 26: 549-555.

27. Blatt NB, Srinivasan S, Mottes T, Shanley MM, Shanley TP. Biology of sepsis: its relevance to pediatric nephrology. Pediatr Nephrol 2014; 29: 2273-2287.

28. Vincent JL, De Backer D. Microvascular dysfunction as a cause of organ dysfunction in severe sepsis. Crit Care 2005; 9(Suppl 4): S9-S12.

29. Sakr Y, Dubois MJ, De Backer D, Creteur J, Vincent JL. Persistent microcirculatory alterations are associated with organ failure and death in patients with septic shock. Crit Care Med 2004; 32: 1825-1831.

30. Venkatachalam MA, Weinberg JM. The tubule pathology of septic acute kidney injury: a neglected area of research comes of age. Kidney Int 2012; 81: 338-340.

31. Maizlin II, Shroyer MC, Perger L, et al. Outcome assessment of renal replacement therapy in neonates. J Surg Res 2016; 204: 34-38.

32. Hakan N, Aydin M, Zenciroglu A, et al. Acute peritoneal dialysis in the newborn period: a 7-year single-center experience at tertiary neonatal intensive care unit in Turkey. Am J Perinatol 2014; 31: 335-338. 